\title{
Evaluation of IR Gas Image System in Open Path Environments
}

\author{
Min Goo Lee ${ }^{1}$, Yong Kuk Park ${ }^{1}$, Kyung Kwon Jung ${ }^{1}$ and Young Jin Kim ${ }^{2}$ \\ ${ }^{1}$ Korea Electronics Technology Institute, ${ }^{2}$ Youngjin Technology co., Ltd \\ emingoo@keti.re.kr
}

\begin{abstract}
This paper describes the tracking system of gas image is to open path to detect the leakage of hazardous gases. The proposed system is able to identify whether or not there are hazardous gases by analyzing the spectrum and absorption's bandwidth of the target gas, and selecting a corresponding band-pass filter. For this work, the gas detectable system which is to use the bandwidth from middle wavelength to long wavelength is designed to apply for the environment using hazardous gases such as methane, ethane, hydrogen sulfide and benzene. IR detector technology is presented to provide a basis for the application of gas-imaged technique. In this paper, our proposed system had been executed the experiments about using gas such as benzene.
\end{abstract}

Keywords: Gas Image Tracking, Infrared Thermal Camera, Spectroscopy, Remote Control

\section{Introduction}

Generally, a detection method of gas leakage, which is managed by high pressure in a large scale of an industrial complex, has been using a thermal image camera based on the temperature difference around the leakage spot. An IR (Infrared) thermal image camera has proved to be the most remarkable technique in that it is able to enhance the capability of maintenance in various industrial parts and detect the mechanical faults in advance. In addition, the IR thermal image camera technique has been causing not only the reduction of a cost but also the improvement of operation-stability, products and processes.

The IR thermal image camera is very effective method to detect the leakage of hazardous gases such as methane, ethane, hydrogen sulfide and benzene and can be used as the part of system in unreachable distance.

Therefore, a large leakage of hazardous gas is able to be detected over distance of hundreds of meters while a small leakage of hazardous gas is able to be detected within the distance of many meters. Moreover, because the IR thermal image camera can detect the leakage of hazardous gas in a mobile vehicle, it can improve the safety and mobility of both inspector and whole factory [1].

In this paper, the IR thermal image camera system to detect the leakage of hazardous gases is suggested. The proposed system is able to identify whether or not there are hazardous gases by analyzing the spectrum and absorption bandwidth of the target gas, and selecting a corresponding band-pass filter. Work, the detectable system of gas which is to use the bandwidth from middle wavelength to long wavelength is designed to apply for the environment using hazardous gases such as methane, ethane, hydrogen sulfide and benzene.

The paper is organized as follows: next section describes a basic concept of IR gas image. Section 3 describes a design and implementation of gas image tracking system. Section 4 describes the results of the indoor and outdoor experiments. Finally, the last section summarizes the results and future works. 


\section{Infrared Characteristics}

The detection of gases by thermal imaging due to absorption, scattering, or emission of IR radiation is a rather new application in the field of infrared thermal imaging.

The proposed system is able to detect the features of strong absorption as using IR camera in the MW (Middle Wavelength) and LW (Long Wavelength) range.

The industrial importance of several gases led to the development of sensitive cameras using narrowband filters in front of the detector. Quantitative analysis of gas concentrations or gas flows is complicated although in the principle also possible.

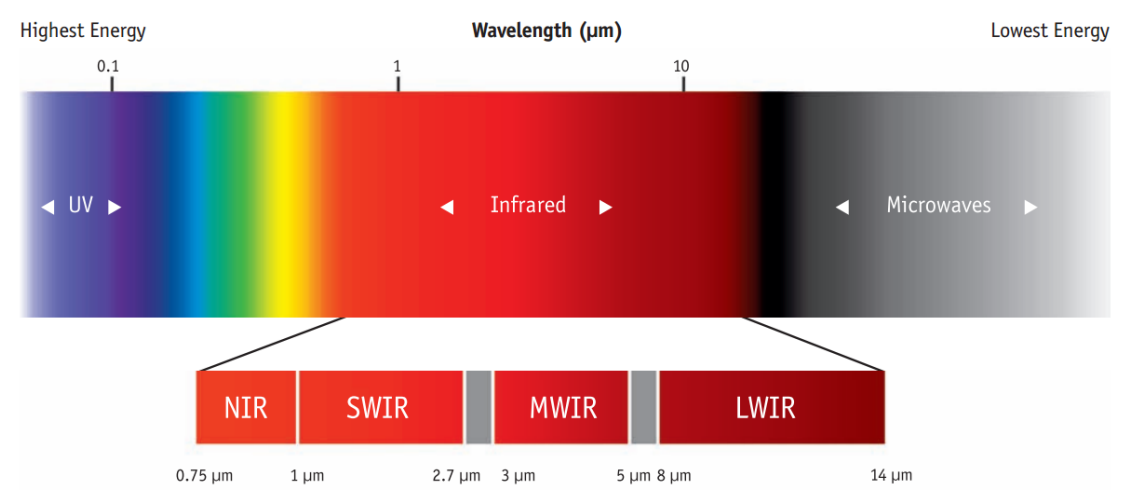

Figure 1. Infrared Spectrum

A further requirement for an instrument to detect gas via the principle of optical absorption is that atmospheric constituents such as water vapor or carbon dioxide do not absorb the optical energy at the same wavelength. The atmosphere is known to have two regions of optical transmission, known as windows, in the infrared. These are the 3 to 5 micron wavelength window in the mid-wave infrared and the 8 to 14 micron wavelength window in the long-wave infrared. Figure 2 shows the optical transmission through the atmosphere for a path length of one kilometer. Figure 2 show that hydrocarbon gas absorption occurs within the MWIR and LWIR optical transmission windows of the atmosphere.

This is indeed fortunate, and has led to the development and widespread use of infrared gas detectors.

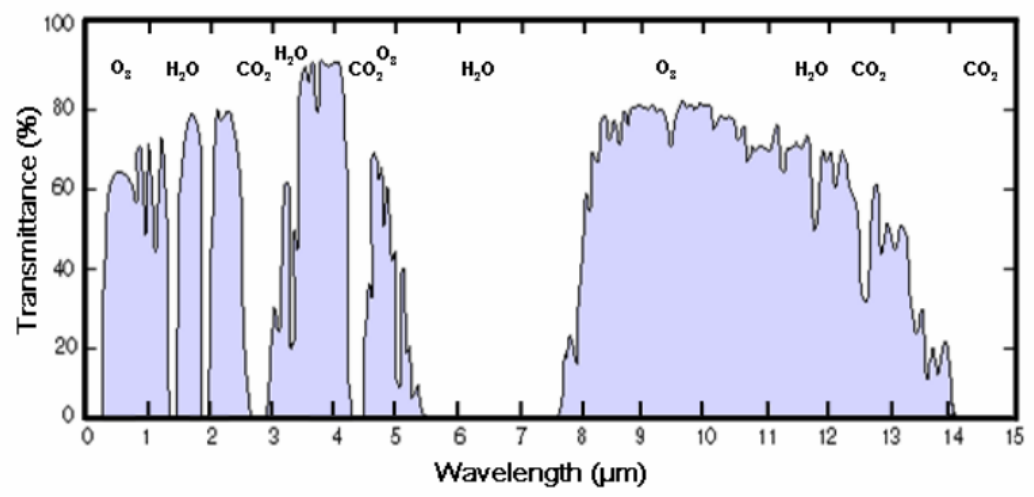

Figure 2. Optical Transmission of the Atmosphere in the Infrared Region

As it is publicly well known, a lot of studies in a spectroscopy were achieved for researching a unique bandwidth of each hazardous gas by which the IR energy is 
absorbed and is not penetrated [3]. Gases have their own characteristic absorption lines in the IR spectrum; VOC's and others have these lines in the region of the spectrum.

The use of a thermal imager as an infrared gas leak detector adjusted to the region of interest will allow the gases to be visualized. Thermal imagers are sensitive to the absorption lines spectrum of the gases and designed to have the optical path sensitivity in correspondence with the gases in the spectrum area of interest. If a component of a system is leaking, the emissions will absorb the IR energy, appearing as smoke black or white.

As shown in Figure 3, gas can operate as band-reject filter by absorbing a unique bandwidth of IR radiated from background. Therefore, a dark image can be seen because the thermal image camera with an appropriate band-pass filter for target gas to be detected cannot measure the unique bandwidth of IR.

The leaking gas temperature differs from the background temperature. The radiation getting to the camera is the background radiation from the background and the radiation from the gas area which obscures the background visualizing the existence of the gas.

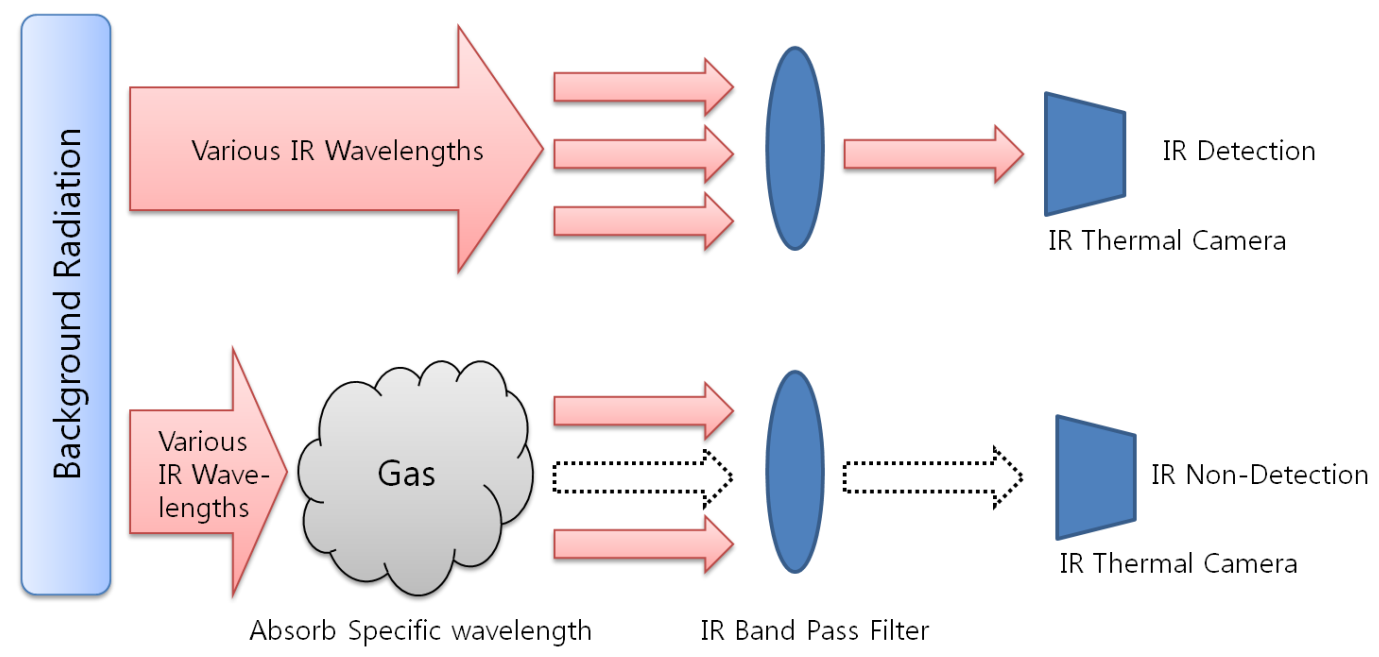

Figure 3. Gas Detection Concepts

In this paper, we focus on Benzene which is dangerous in daily life. The absorption bands of Benzene lay around wavelength of $3.3 \mathrm{um}, 6.7 \mathrm{um}, 9.5 \mathrm{um}$, and $15 \mathrm{um}$ in Figure 4. The wavelength $9.5 \mathrm{um}$ and $15 \mathrm{um}$ are within the spectral range of LW IR cameras.

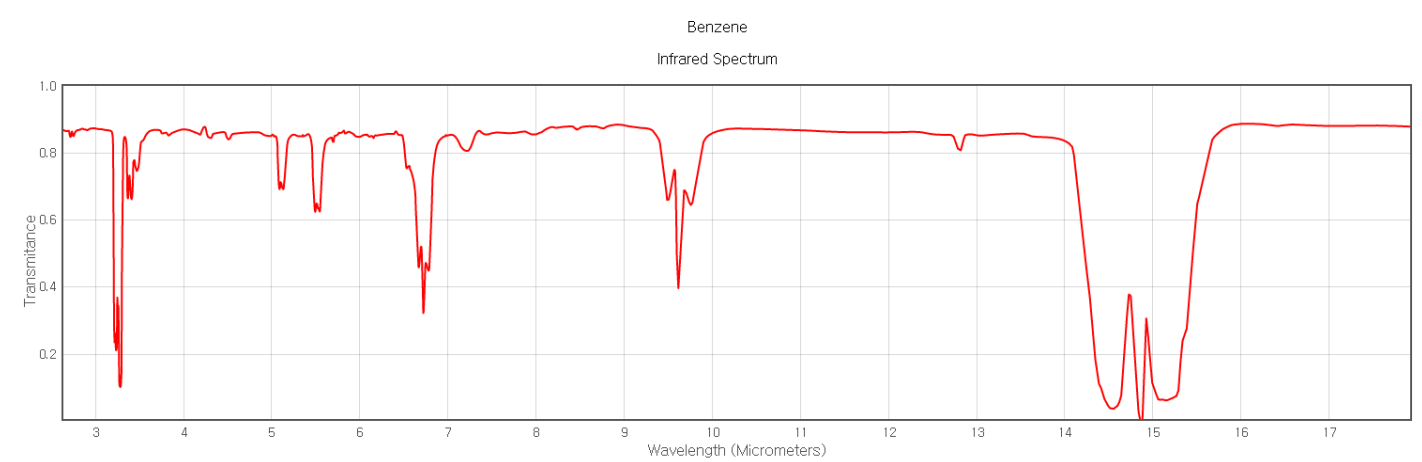

Figure 4. IR spectrum of Benzene [4] 


\section{IR Gas System Overview}

The gas detection system suggested in this paper is shown in Figure 5. The system comprise IR camera, IR band pass filter, CCD camera and Pan/Tilt controller.

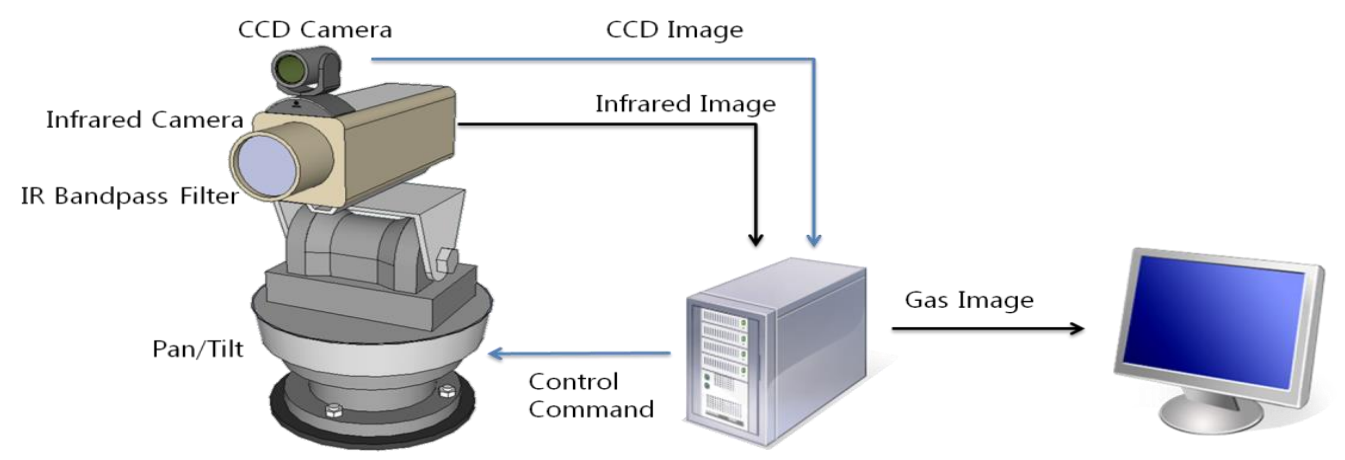

Figure 5. System Configuration

Figure 6 shows the internal function blocks of designed IR thermal image camera and Figure 7 represents the implemented hardware blocks as well.

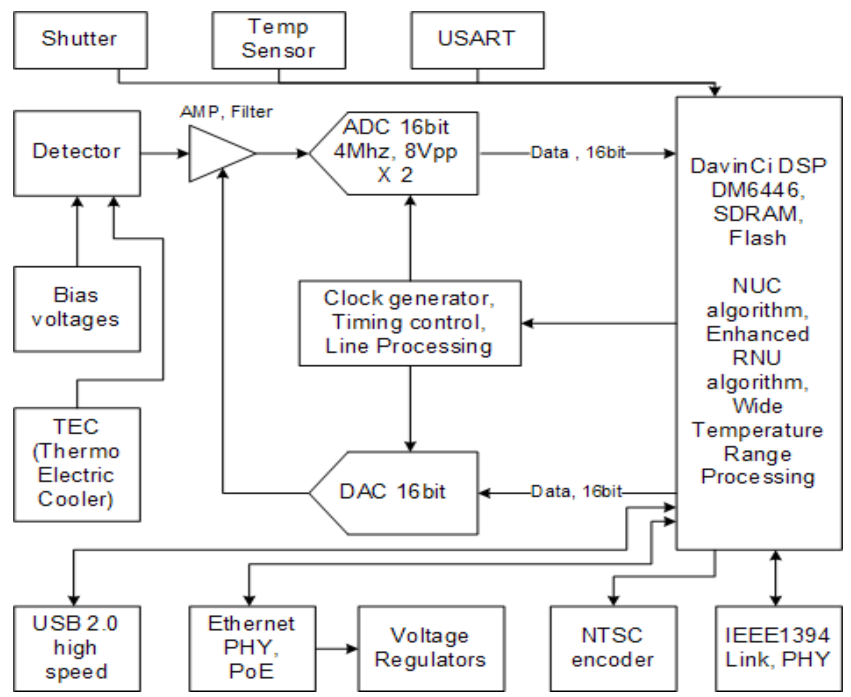

Figure 6. Block Diagram of IR Camera

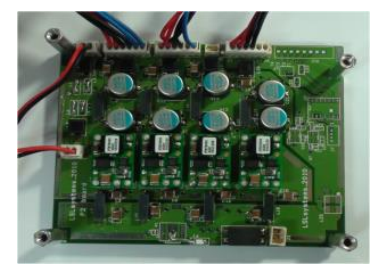

(a) Power Supply

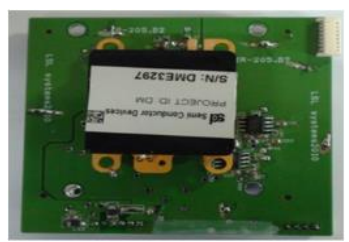

(d) Detector

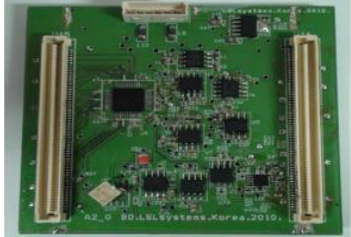

(b) ADC

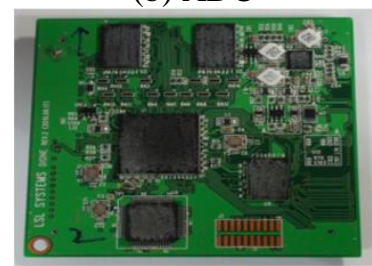

(e) DSP

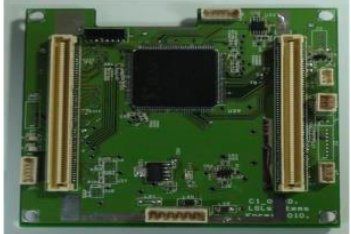

(C) CPLD

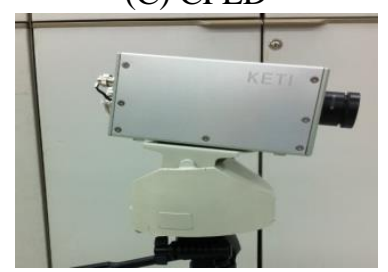

(f) IR Camera

Figure 7. Implemented IR Thermal Image Camera 


\section{Experiments}

As shown in Figure 8, the experiments to detect the hazardous gas were performed with benzene in the lab environment. For a safety of this experiment, an exhaustion were fully carried out during the test and the hazardous gas was not continuously emitted but instantly emitted within 1 or 2 seconds. Figure 9 represented the measurement results of hazardous benzene gas through image process by monitoring software.

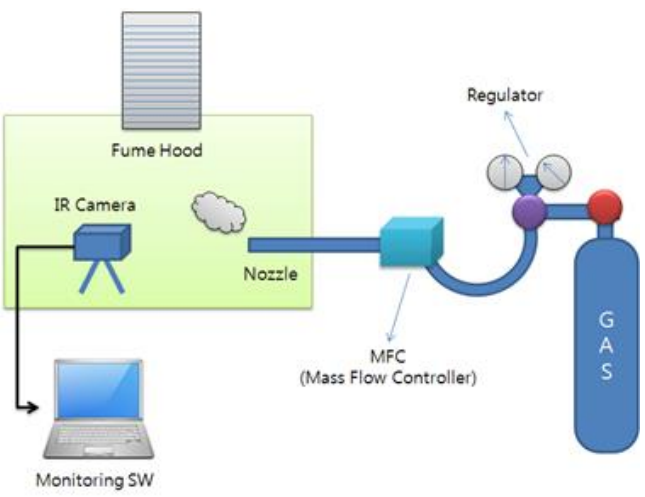

(a) Block Diagram

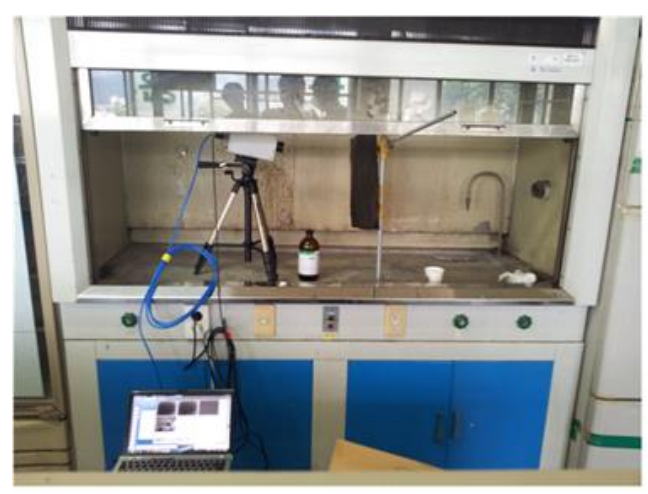

(b) Experimental Setup

Figure 8. Test Setup in the Lab

Figure 9 represented the measurement results of hazardous benzene gas through image process by monitoring software.

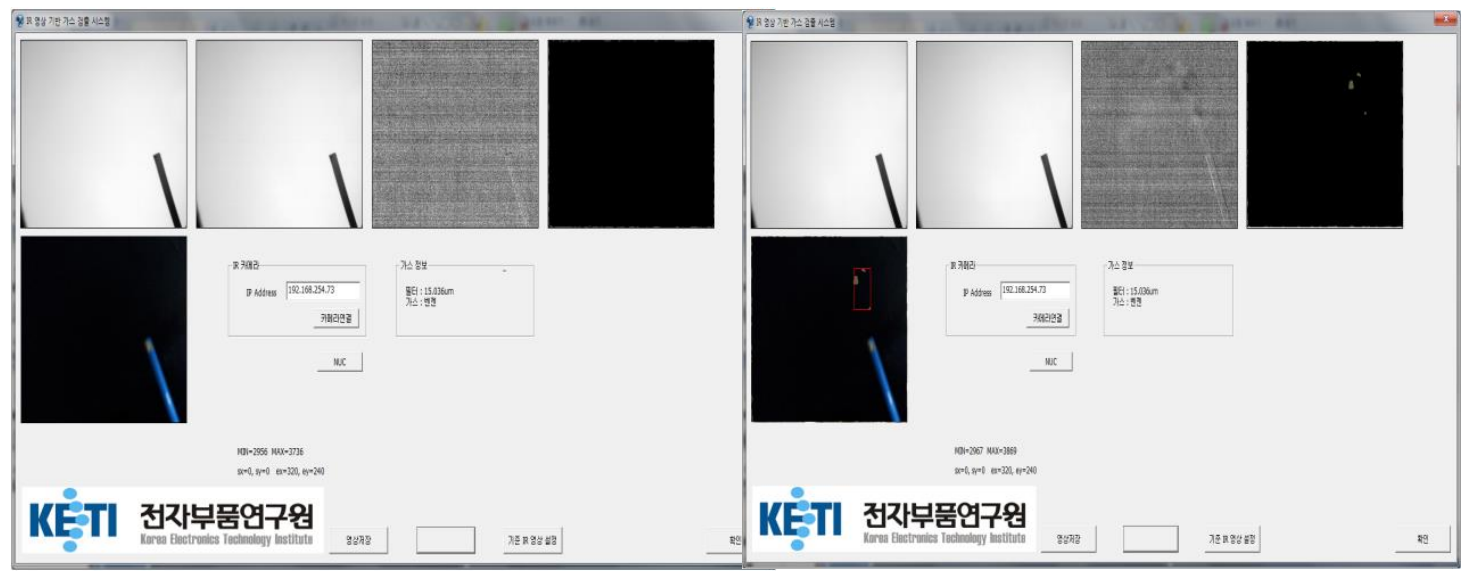

(a) With No Benzene Gas

(b) With Benzene Gas

Figure 9. Test Results

Most applications for a gas imaging system are out of doors, where the local wind field acts to disperse the gas. As a demonstration, we tested the proposed system outside by imaging benzene as shown in Figure 10. As before, we created movies using the recorded images. Figure 11 shows image taken from the movies. The results show that the system is capable of producing useful images of such sources in real-world conditions. 

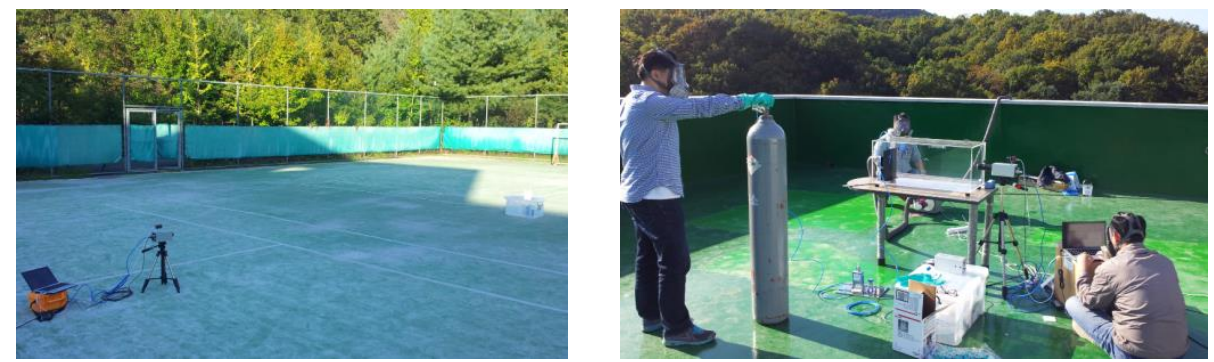

Figure 10. Test Setup in Outdoor Environment

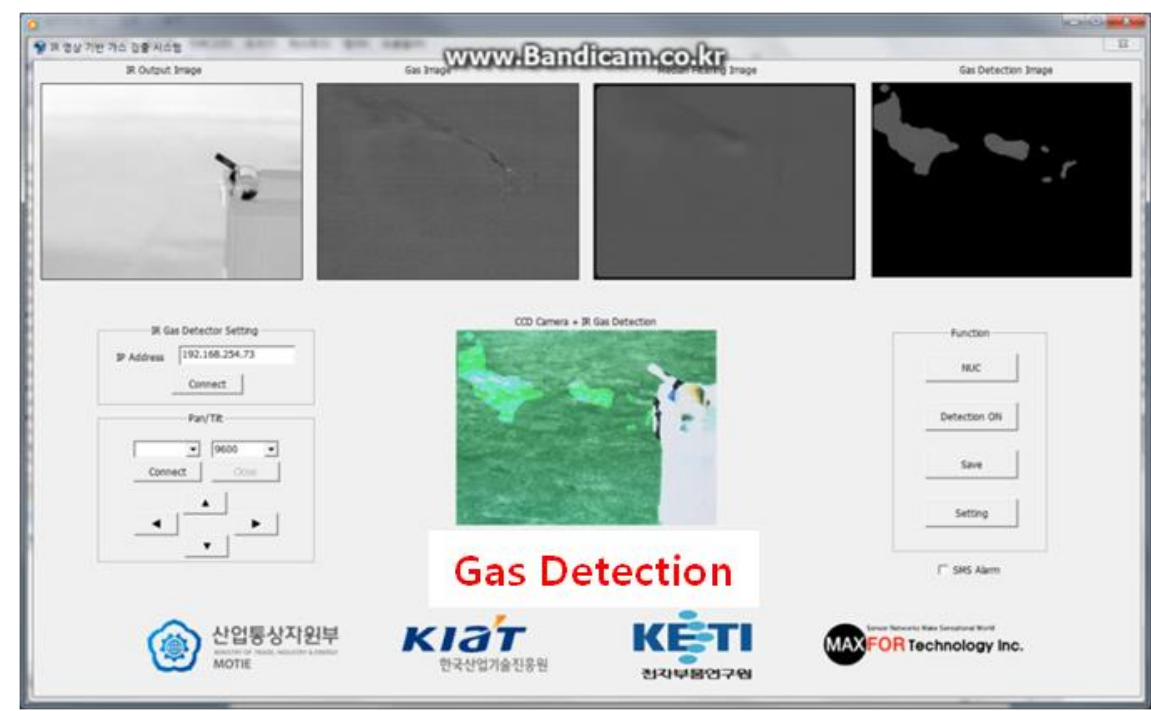

Figure 11. Test Results

\section{Conclusions}

This paper suggested the gas detection system using IR thermal image camera. The proposed system is able to detect the corresponding bandwidth of IR by selecting a unique bandwidth of each hazardous gas by which the IR energy is absorbed and is not penetrated, and using image process technique. In addition, it is possible to visualize the quantity of IR and calculate the distribution area of hazardous gas through the rotary filter bank. The IR thermal image camera including filter operator and CCD camera was designed to detect the hazardous gases such as methane, ethane, hydrogen sulfide and benzene which are meaningful in both middle wavelength (3 5um) and long wavelength (8 12um).

\section{Acknowledgments}

This research was supported by Business for Cooperative R\&D between Industry, Academy, and Research Institute funded Korea Small and Medium Business Administration in 2014 [No. C0213232].

\section{References}

[1] Gas detection handbook - Flir Systems, http://www.flir.com/.

[2] M. Vollmer and K.-P. Möllmann, "Infrared Thermal Imaging: Fundamentals, Research and Applications", Wiley-VCH, Weiheim Germany, (2010).

[3] J. Sandsten, H. Edner and S. Svanberg, "Gas visualization of industrial hydrocarbon emissions", (2004), vol. 12, no. 7, pp. 1443-1451.

[4] NIST Benzene, http://webbook.nist.gov/cgi/cbook.cgi?Name=benzene\&cIR=on. 


\section{Authors}

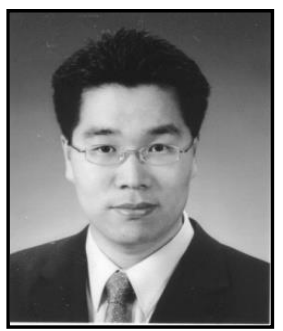

Min Goo Lee received the BS and MS degrees in Electronics Engineering from Sogang University, Seoul, Korea in 2000, 2004 respectively. $\mathrm{He}$ is currently a senior researcher in U-embedded Convergence Research Center at Korea Electronics Technology Institute (KETI). His research interests include wireless sensor networks, energy management in smart home and IR gas imaging.

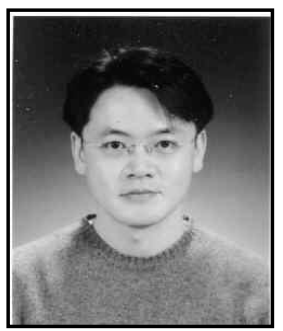

Yong Kuk Park received the BS and MS and PhD degrees in Electronics Engineering from Chung-Ang University, Seoul, Korea in 1994, 1996, 2012 respectively. He is currently a senior researcher in U-embedded Convergence Research Center at Korea Electronics Technology Institute (KETI). His research interests include Energy Management, virtual power plant and IR gas imaging.

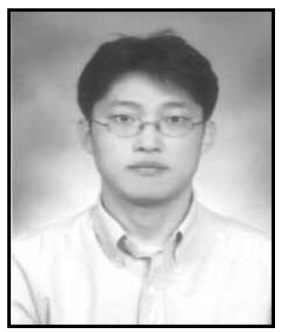

Kyung Kwon Jung received the BS, MS and $\mathrm{PhD}$ degrees in Electronics Engineering from Dongguk University, Seoul, Korea in 1998, 2000 and 2003 respectively. He is currently a research engineer in U-embedded Convergence Research Center at Korea Electronics Technology Institute (KETI). His research interests include wireless sensor networks, embedded system and IR gas imaging.

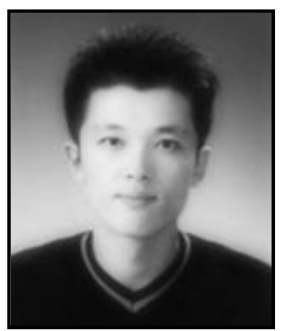

Young Jin Kim received the BS and MS degrees in Physics and Mobile Communication Engineering from Sung Kyun Kwan University, Seoul, Korea in 2001 and 2011 respectively. He is currently a research engineer and founder at Youngjin Technology co., Ltd. His research and business interests include wireless sensor networks, embedded system, environment monitoring system and devices and Energy monitoring. 
International Journal of Advanced Science and Technology Vol.83 (2015) 